Cite this: Phys. Chem. Chem. Phys., 2013, 15, 9575

Received 9th January 2013, Accepted 10th April 2013

DOI: $10.1039 /$ c3cp00109a

www.rsc.org/pccp

\section{Size-dependent Auger spectra and two-hole Coulomb interaction of small supported Cu-clusters}

\author{
S. Peters, ${ }^{a b}$ S. Peredkov, ${ }^{a b}$ M. Neeb, ${ }^{* a b}$ W. Eberhardt ${ }^{a b}$ and M. Al-Hada ${ }^{c}$
}

\begin{abstract}
Auger $\left(L_{3} M_{4,5} M_{4,5}\right)$ and $X$-ray photoionization spectra $(2 p, 3 d)$ of mass-selected $C_{N}$-clusters supported by a thin natural silica layer are presented in the size range $N=8-55$ atoms per cluster. The Auger spectra of all clusters are shifted to a lower kinetic energy with respect to the spectrum of the bulk. Furthermore the Auger energy decreases systematically with decreasing cluster size. The binding energies of the $2 p$ and $3 d$ valence states are higher than the corresponding bulk values. Using the energy of the Auger main line, the corresponding core hole peak and the centroid of the selfconvoluted $3 \mathrm{~d}$ valence band the on-site Coulomb interaction energy $U_{\mathrm{dd}}$ of the two-hole final state as a function of cluster size has been determined.
\end{abstract}

\section{Introduction}

Core-level photoelectron spectroscopy is a surface sensitive and element specific tool which can be applied to study highly diluted samples such as free and deposited clusters by X-ray photoelectron spectroscopy (XPS), ${ }^{1-9} \mathrm{X}$-ray absorption spectroscopy $(\mathrm{XAS})^{10-12}$, extended X-ray absorption fine structure analysis (EXAFS) ${ }^{13}$ grazing-incidence small-angle X-ray scattering (GISAXS), ${ }^{14}$ X-ray magnetic circular dichroism (XMCD) ${ }^{15-17}$ and Auger electron spectroscopy (AES). ${ }^{18}$ In particular AES and XPS provide information on the initial and final states such as chemical effects, charge transfer, core-hole screening, core-hole binding energies, and electron correlation including final-state term splitting and on-site electron-electron interaction. Regarding electron correlation Auger spectra have been used to obtain valuable information on the effective Coulomb interaction of two 3d holes in transition metals and their compounds such as oxides and cuprates. ${ }^{19,20}$ Information on the localized versus itinerant behaviour of the $3 \mathrm{~d}$ electrons can thus be gained. ${ }^{19}$ Moreover by comparing the effective Coulomb interaction with the one-electron valence band width information on the Mott-Hubbard behaviour of d-band metals is obtained. ${ }^{19}$ In general, the Coulomb interaction in bulk metals is substantially reduced with respect to the atomic value due to extra-atomic

\footnotetext{
${ }^{a}$ Helmholtz-Zentrum Berlin, Wilhelm-Conrad-Röntgen-Campus Adlershof, Elektronenspeicherring BESSY II, Albert-Einstein-Straße 15, 12489 Berlin, Germany. E-mail: matthias.neeb@helmholtz-berlin.de

${ }^{b}$ Technische Universität Berlin, IOAP, Strasse des 17. Juni 135, 10623 Berlin, Germany

${ }^{c}$ Department of Physics, College of Education and Linguistics, University of Amran, Amran, Yemen
}

contributions such as polarization and screening. In copper, for instance, the free-atom value of the on-site two-hole Coulomb correlation amounts to $27 \mathrm{eV}$ while the corresponding value of the bulk is merely $7-8 \mathrm{eV} \cdot{ }^{19,21}$ In the present paper we study the evolution of the d-hole Coulomb interaction energy of $\mathrm{Cu}$ as a function of cluster size. Questions of interest are the size scale on which the Coulomb interaction approaches the bulk limit and whether the change proceeds smoothly or discontinuously as a function of size. Note that copper is considered as a textbook example for studying d-band Coulomb correlation by LMM Auger spectroscopy due to its $\mathrm{d}^{8}$ final state configuration, well separated initial core-hole states $\mathrm{L}_{2}$ and $\mathrm{L}_{3}$ and well understood LMM Auger spectrum. ${ }^{19,22}$ The clusters are supported by a thin Si-oxide layer which only moderately interacts with the cluster and is frequently used as a model support in studies of the catalytic behaviour of clusters.

\section{Experimental}

Small $\mathrm{Cu}_{N}$-clusters were synthesized using a home-built magnetron sputtering source and mass-filtered by a magnetic sector field analyser $^{23}$ at a mass resolution of $m / \Delta m \sim 100$. A typical mass spectrum is shown in Fig. 1. In brief, a mass-filtered cluster beam is softly landed onto a biased p-doped $\mathrm{SiO}_{2} / \mathrm{Si}(100)$-substrate $(<1 \mathrm{eV}$ per atom) through a $6 \mathrm{~mm}$ final aperture under UHV conditions $\left(<1 \times 10^{-9} \mathrm{mbar}\right)$. Typical mass-filtered cluster ion currents range from 10-100 pA depending on the cluster size. Deposition times of 10-30 minutes were used to obtain a total coverage density of $\sim 10^{13}$ atoms per $\mathrm{cm}^{2}$ (ca. 1\% atomic surface coverage). The deposition area has been monitored using a position sensitive MCP/CCD device. Using a battery-driven mobile UHV chamber 


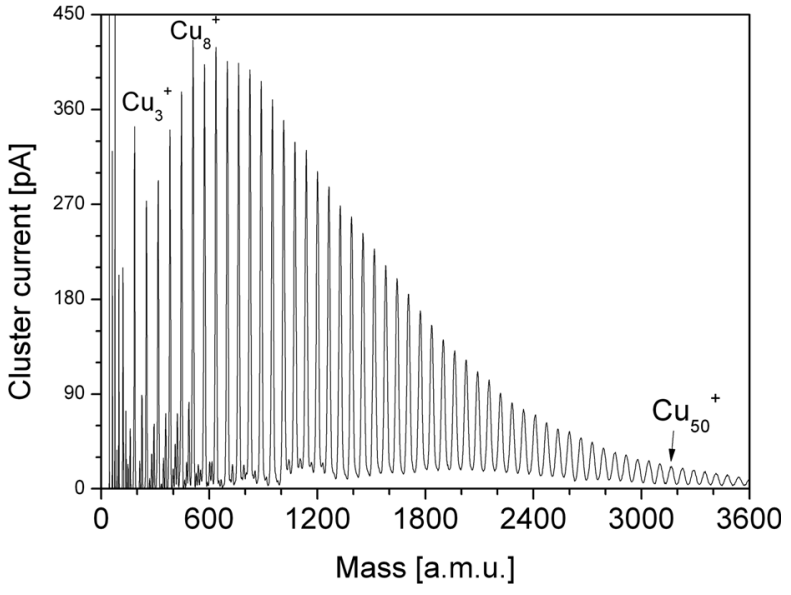

Fig. 1 Magnetic sector field mass spectrum of $\mathrm{Cu}_{N}$-cluster cations recorded at a resolution of $m / \Delta m \sim 100$. During deposition the current is ca. 5 times lower due to soft-landing conditions.

the cluster-covered samples were transferred and lock loaded into the photoemission endstation of the PM4 dipole beamline at BESSY $\left(<10^{10}\right.$ photons per $\mathrm{s}$, linear polarisation $)$ without interrupting the vacuum $\left(p<5 \times 10^{-9} \mathrm{mbar}\right)$. Auger spectra of different clusters between $\mathrm{Cu}_{8}$ and $\mathrm{Cu}_{55}$ have been recorded $(N=$ $8,10,13,19,26,35,38,50,55)$ using a photon energy $h \nu=980 \mathrm{eV}$ for recording $\mathrm{L}_{3} \mathrm{MM}$ Auger spectra, $h \nu=1150 \mathrm{eV}$ for recording $2 \mathrm{p}$ XPS spectra and $h \nu=200 \mathrm{eV}$ for recording valence band spectra. A commercial electron spectrometer (Scienta SES 100) has been used for measuring the kinetic energy of Auger and photoelectrons. Each cluster spectrum has been recorded within $1-2 \mathrm{~h}$. The cluster spectra are compared to the Auger and XPS spectra of a sputtered copper single crystal. All cluster spectra have been recorded with the same experimental resolution as the bulk spectrum. The measured kinetic energy of each cluster sample has been calibrated with respect to the Si 2p peak of the substrate which is in proper electrical contact with the spectrometer.

\section{Results and discussion}

Fig. 2 shows the $\mathrm{L}_{3} \mathrm{M}_{4,5} \mathrm{M}_{4,5}$ Auger spectra of supported $\mathrm{Cu}_{N^{-}}$-clusters with $N=8,10,13,19,26,35,38,50,55$ atoms in comparison to the bulk Auger spectrum. The main Auger multiplets $\left({ }^{1} \mathrm{~S},{ }^{1} \mathrm{G},{ }^{3} \mathrm{~F}\right)$ of the bulk spectrum are labelled according to the notation of Antonides et al. ${ }^{19}$ The excitation energy of $980 \mathrm{eV}$ is well above the $\mathrm{L}_{3}$ core ionization threshold where no photon energy dependence of the Auger transitions is observed. Therefore fully opened monochromator slits were used for maximum photon flux $\left(10^{10}-10^{11} \mathrm{~s}^{-1}\right)$ to record the so-called "sudden-limit" Auger spectrum. The experimental resolution of the electron analyser was set to $1.6 \mathrm{eV}$ as a compromise of high transmission and sufficient resolution.

While the overall spectral shape of the cluster Auger spectra is similar to the bulk spectrum the former are considerably shifted to lower kinetic energies as seen in Fig. 2. The peak maximum ( $\left({ }^{1} \mathrm{G}\right.$ multiplet) of the bulk is located at $918.4 \mathrm{eV}$ while the maximum of the cluster Auger spectra is clearly reduced
A

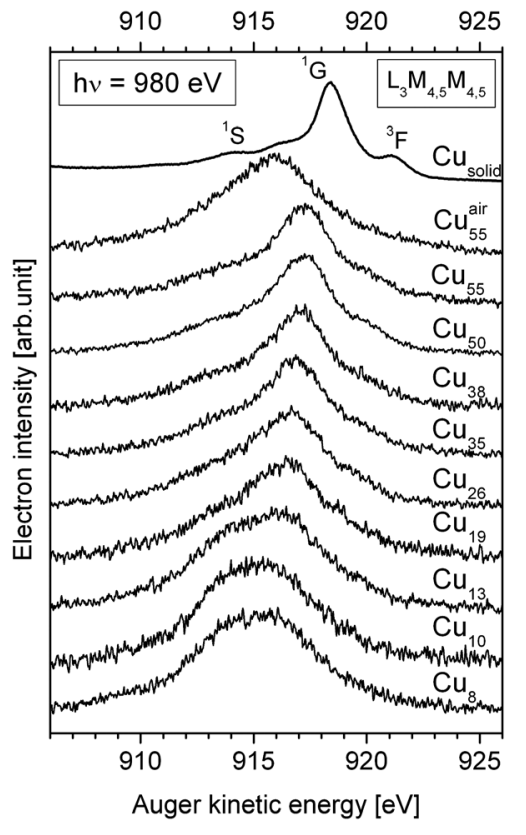

B

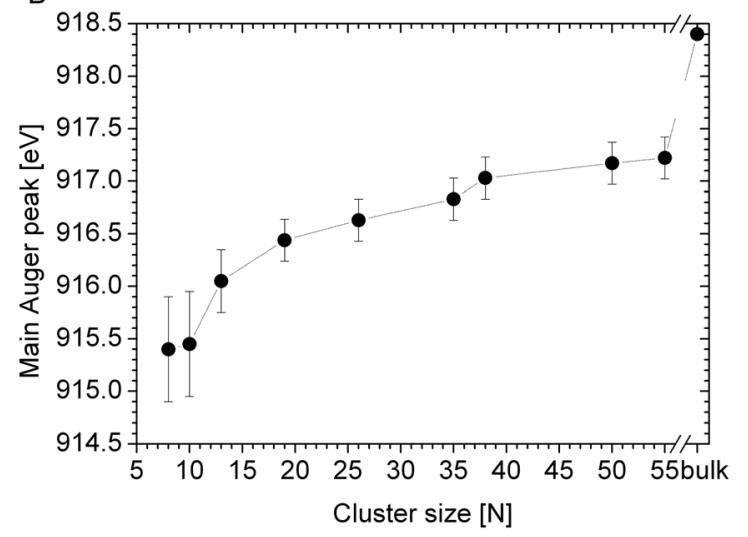

Fig. 2 (A) $L_{3} M_{4,5} M_{4,5}$ Auger spectra of $C_{N^{-}}$-Clusters $(N=8-55)$ supported by a thin silica layer naturally grown on a $\mathrm{Si}$ wafer $\left(\mathrm{SiO}_{2} / \mathrm{Si}\right)$. The cluster spectra are compared to the bulk Auger spectrum of a sputtered and annealed single crystal $\mathrm{Cu}(111)$ taken under the same experimental conditions (top). A kinetic energy resolution of $1.6 \mathrm{eV}$ has been used to record the Auger spectra at a photon energy of $980 \mathrm{eV}$ (sudden limit Auger spectra). The notation of the bulk Auger transitions is taken from ref. 19. The weak Auger feature between feature $S$ and $G$ belongs to a Coster-Kronig preceded Auger transition $\left(L_{2} L_{3} M_{4,5}\right)$ of the initial $2 p_{1 / 2}$ core-hole state. ${ }^{22}$ An Auger spectrum of atmospherically oxidised $\mathrm{Cu}_{55}$ is additionally shown to demonstrate the effect of oxidation. (B) Kinetic energy of the main Auger peak $\left({ }^{1} \mathrm{G}\right)$ as a function of cluster size $(N)$ used to evaluate the Coulomb interaction energy.

ranging between 915 and $917 \mathrm{eV}$ (Fig 2). Note that the kinetic energy gradually decreases with decreasing cluster size as obvious from Fig. $2 \mathrm{~b}$ which shows the energy of the main Auger transition $\left({ }^{1} \mathrm{G}\right)$ as a function of size. While the Auger shift (energy difference between bulk and cluster) of small clusters amounts to more than $3 \mathrm{eV}$ the largest measured cluster $\mathrm{Cu}_{55}$ still has an Auger shift of $1.5 \mathrm{eV}$.

Also the shape and envelope of the Auger spectra vary with size. The main line narrows with increasing cluster size. Moreover, the intensity at kinetic energies lower than the main peak is considerably enhanced in the spectra of small clusters 
$(N \leq 13)$. This additional Auger intensity is attributed to oxidation-like initial state modifications of small clusters due to their interaction with the silica support. In fact the Auger kinetic energy shifts to lower values upon oxidation as shown for the bulk phase of $\mathrm{Cu}$ and as shown here by the spectrum of atmospherically oxidised $\mathrm{Cu}_{55}$ in the top panel of Fig. 2A. The shift to lower kinetic energies can be explained by partial depletion of the $3 \mathrm{~d}$ band due to an initial-state charge transfer from copper to oxygen as discussed for oxidised copper bulk samples. ${ }^{20,24}$ Note that mainly each atom of a small cluster is in contact with the supporting oxide layer while for large compact clusters the contact area between the support and the cluster is reduced. Therefore the cluster-surface interaction, i.e. charge transfer due to copper-oxygen interaction, is more emphasized in the spectra of small clusters than larger ones. Also the XPS binding energy exhibits the largest shift for these clusters (see Fig. 3B).

The Auger kinetic energy is generally given as a difference between the initial core-hole state and the doubly-ionized final state. In particular, the energy of the $\mathrm{L}_{3} \mathrm{M}_{4,5} \mathrm{M}_{4,5}$ Auger transitions $E_{\text {kin }}\left(\mathrm{L}_{3} \mathrm{M}_{4,5} \mathrm{M}_{4,5}\right)$ corresponds to the energy difference between the $2 \mathrm{p}_{3 / 2}{ }^{-1}$ core-hole state $E_{\mathrm{bin}}\left(2 \mathrm{p}_{3 / 2}\right)$ and the valence ionized $3 \mathrm{~d}^{-2}$ final state $E_{\text {bin }}(3 \mathrm{~d})$ including the d-d Coulomb interaction energy $U_{\mathrm{dd}}$ of the double vacancy.

$$
\begin{aligned}
& E_{\text {kin }}\left(\mathrm{L}_{3} \mathrm{M}_{4,5} \mathrm{M}_{4,5}\right)=E_{\text {bin }}\left(2 \mathrm{p}_{3 / 2}\right)-2 E_{\text {bin }}(3 \mathrm{~d})-U_{\mathrm{dd}} \\
& \Leftrightarrow U_{\mathrm{dd}}=E_{\text {bin }}\left(2 \mathrm{p}_{3 / 2}\right)-E_{\text {kin }}\left(\mathrm{L}_{3} \mathrm{M}_{4,5} \mathrm{M}_{4,5}\right)-2 E_{\text {bin }}(3 \mathrm{~d})
\end{aligned}
$$

Thus by combining the binding energy of the core and valence levels with the Auger kinetic energy the above relation allows determining the $\mathrm{Cu} 3 \mathrm{~d}$ two-hole correlation energy $U_{\mathrm{dd}}$. In the case of a photoionized cluster a possible final-state Coulombcharging has to be considered due to a delayed neutralisation of the photohole as originally discussed by G. Wertheim. ${ }^{25}$ The electrostatic interaction between the emitted electron and the remaining hole $E_{\text {Coul }}$ will affect the energy of the Auger electron and photoelectron in addition to the ordinary initial and final state effects. In case of clusters, this extra energy term has to be considered in eqn (1) used to evaluate $U_{\mathrm{dd}}$ from the measured binding and kinetic energy of the photoelectron and Auger electron, respectively. According to a final state consideration (Fig. 5) the measured energy difference $U_{\mathrm{dd}}^{\text {meas }}$ between the XPS binding energy, Auger energy and two times the valence band maximum of a photoionized cluster differs by two times the Coulomb energy with respect to the bulk metal:

$U_{\mathrm{dd}}^{\text {meas }} \equiv U_{\mathrm{dd}}^{\mathrm{cl}}+2 E_{\text {Coul }}=E_{\text {bin }}^{\mathrm{cl}}\left(2 \mathrm{p}_{3 / 2}\right)-E_{\text {kin }}^{\mathrm{cl}}\left(\mathrm{L}_{3} \mathrm{M}_{4,5} \mathrm{M}_{4,5}\right)-2 E_{\text {bin }}^{\mathrm{cl}}(3 \mathrm{~d})$

Eqn (2) is derived from the fact that the electrostatic energy of a singly charged sphere with radius $R$ and charge $q$ is proportional to $q^{2} / R\left(1 E_{\text {Coul }}\right) .^{25,26}$ Consequently, the energy of a doubly charged sphere is proportional to $(2 q)^{2} / R$ which corresponds to 4 times the Coulomb energy of a singly charged sphere $\left(4 E_{\text {Coul }}\right)$ as pointed out by ref. 25 . For supported clusters the Coulomb energy might be reduced due to extra-atomic screening by the substrate. Note that the amount of substrate
A

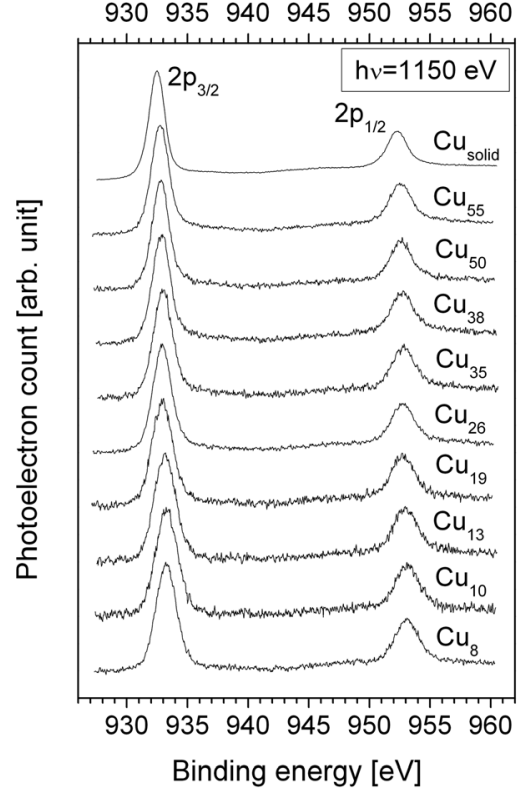

B

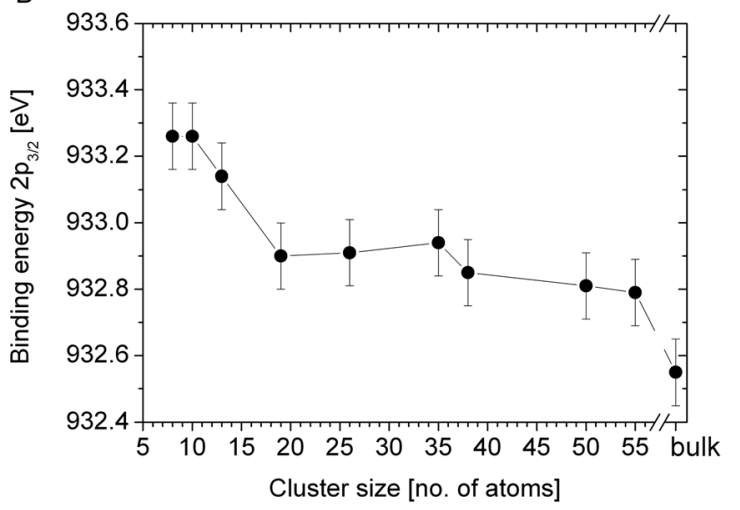

Fig. 3 (A) $2 p$ XPS spectra of $\mathrm{Cu}_{N}$-clusters $(N=8-55)$ supported by a thin silica layer naturally grown on a $\mathrm{Si}$ wafer $\left(\mathrm{SiO}_{2} / \mathrm{Si}\right)$. The cluster spectra are compared to the bulk XPS spectrum of a sputtered and annealed single crystal $\mathrm{Cu}(111)$ taken under the same experimental conditions (top). A total experimental energy resolution of $1 \mathrm{eV}$ has been used to record the XPS spectra at a photon energy of $1150 \mathrm{eV}$. (B) Binding energy of the main $2 p_{3 / 2}$ peak as a function of cluster size $(N)$ used to evaluate the effective Coulomb interaction energy.

screening depends on the conductivity of the substrate and the interaction-strength between the cluster and the substrate.

The single-hole binding energies have been derived from the inner-shell and valence-band photoelectron spectrum, respectively. The $2 p$ XPS spectra of the clusters are shown in Fig. 3 which have been measured at $h \nu=1150 \mathrm{eV}$ and a total experimental resolution of $1 \mathrm{eV}$, i.e. larger than the core-hole lifetime broadening of the $\mathrm{Cu} 2 \mathrm{p}_{3 / 2}$ shell $(\Gamma=0.6 \mathrm{eV}$ (ref. 27)). As seen in Fig. 3A the XPS peaks of the clusters are shifted to a higher binding energy with respect to the bulk peaks and are considerably broadened. The XPS shift ranges between $0.3\left(\mathrm{Cu}_{55}\right)$ and $0.9 \mathrm{eV}\left(\mathrm{Cu}_{8}\right)$ for both spin-orbit components. The reason for the systematic high energy XPS shift and broadening is mainly attributed to inhomogeneous broadening of inequivalent cluster atoms as well as final state effects such as reduced core-hole screening and Coulomb interaction between 
the photoelectron and the ionized cluster. ${ }^{28,29}$ Moreover extra atomic charge neutralisation by the substrate according to the dynamic liquid drop model ${ }^{30}$ which partially compensates the final state Coulomb shift must be considered for a quantitative interpretation as recently discussed for the $4 \mathrm{f}$ XPS peak of small $\mathrm{Au}$ clusters on silica. ${ }^{9}$ A thorough analysis of the XPS peak shape and analysis of the shift, however, are not the main purpose of this paper and will be done once XPS spectra with a resolution better than the core-hole lifetime are available. Here we are mostly interested in the absolute energy of the $2 \mathrm{p}_{3 / 2}$ peak which is straightforwardly determined from the spectra and compiled in Fig. 3B. These values are used later on to evaluate $U_{\mathrm{dd}}^{\mathrm{cl}}$. Note that the XPS shift is much smaller than the corresponding Auger shift due to the fact that the binding energy shift of the valence shell as well as the two-hole Coulomb interaction additionally contributes to the Auger shift. ${ }^{25}$

Except for the continuous energy shift the XPS spectra reveal no distinct differences such as high energy asymmetry or discontinuous energy shift when changing from smaller to larger clusters $(N>13)$ in contrast to the Auger spectra for which a distinct change in the spectral shape is observed for small clusters (Fig. 2A). This spectral change is here tentatively attributed to oxidation-like interaction between the cluster and the silica support which shifts the Auger kinetic energy to lower energies. The XPS spectra of the cluster indicate that the interaction between the cluster and the silica layer leads to $\mathrm{Cu}^{1+}$ rather than fully oxidised $\mathrm{Cu}^{2+}$ ions. We conclude this from the comparison with the XPS spectrum of the $\mathrm{Cu}_{2} \mathrm{O}$ bulk phase which reveals almost no energy shift $(\sim 0.1 \mathrm{eV})$ with respect to the metal bulk phase of pristine $\mathrm{Cu}$. $\mathrm{CuO}$, in contrast, shows a strong XPS shift $(\sim 1 \mathrm{eV})$ with respect to the metal bulk phase. $^{20,24}$ Moreover very strong shake-up satellite peaks behind the $2 p_{3 / 2}$ and $2 p_{1 / 2}$ main lines are observed for fully oxidised copper atoms in $\mathrm{CuO} .^{20,24}$ Such oxide-induced satellite peaks are not observed in the XPS spectra of the supported cluster which gives further evidence that the cluster-substrate interaction does not lead to oxidic clusters. The contact with the oxide layer rather induces a partial charge transfer resulting in $\mathrm{Cu}^{1+}$ ions of $\mathrm{d}^{10}$ configuration, in particular for those atoms which are in contact with the support.

As discussed in ref. 20 and 21 the energy of twice the valence band maximum ( $\left.2 E_{\text {bin }}(3 \mathrm{~d})\right)$ is most reliably taken from the selfconvoluted single-hole valence band intensity distribution. Such a self-convoluted single-hole valence band spectrum corresponds to the correlation-free two-hole binding energy spectral distribution of the valence band. The peak maximum of the self-convoluted spectrum is taken as the hypothetical mean energy to remove two electrons independently from the valence band. The corresponding valence band spectrum and the numerically self-convoluted valence band spectrum are shown in Fig. 4. The X-ray induced valence band spectra have been recorded with $h \nu=200 \mathrm{eV}$ and a total experimental resolution of $0.8 \mathrm{eV}$. The valence band spectra of the lowdensity cluster samples are superimposed by the signal of the $\mathrm{Si} / \mathrm{SiO}_{2}$ valence band. Therefore the valence band of the clean support (measured for each sample at a position without the

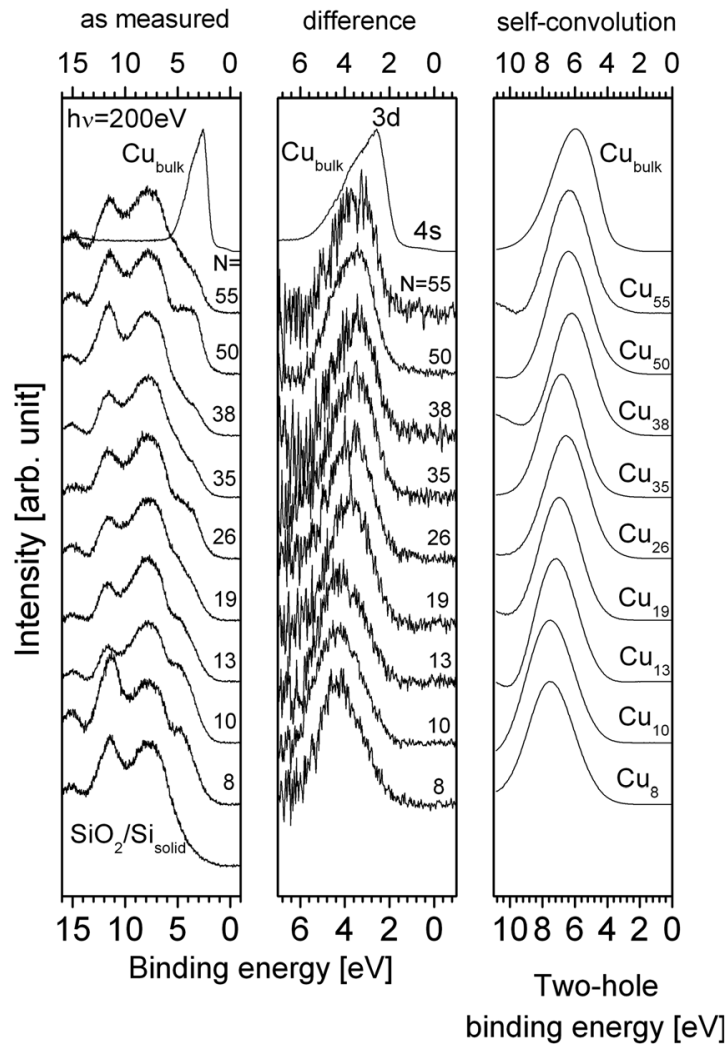

Fig. 4 Left: X-ray induced valence band spectra of $\mathrm{Cu}_{N^{-}}$-clusters $(N=8-55)$ supported by a thin silica layer naturally grown on a Si wafer $\left(\mathrm{SiO}_{2} / \mathrm{Si}\right)$. The cluster spectra are compared to the valence spectrum of a sputtered and annealed single crystal $\mathrm{Cu}(111)$ taken under the same experimental conditions (top). A total experimental energy resolution of $0.8 \mathrm{eV}$ has been used to record the valence band spectra at a photon energy of $200 \mathrm{eV}$. The Fermi energy is calibrated by means of the $4 \mathrm{~s}$ valence band of Cu-bulk (turning point). The cluster specific valence band is seen at binding energy $<5 \mathrm{eV}$. The valence band of the clean support is shown at the bottom of the panel. (centre) Subtracted valence band spectrum showing the cluster specific spectral intensity below $5 \mathrm{eV}$. (right) Self-convoluted single-hole valence band spectrum exhibiting the twohole spectral distribution of the valence shell.

cluster signal) has been subtracted from the superimposed valence band spectrum. The binding energy refers to the Fermi energy of the Cu-bulk. Note that the intensity above a binding energy of $5 \mathrm{eV}$ is dominated by the valence band of Si. Below $5 \mathrm{eV}$ the $\mathrm{Cu}$ valence band is clearly enhanced with respect to the leading edge of the Si-valence band. Upon subtraction of the $\mathrm{Si}$ valence band from the superimposed valence band spectra the remaining intensity can be interpreted as the main part of the $3 \mathrm{~d}$ valence spectrum of the clusters. The spectral shape is similar to the $3 \mathrm{~d}$ band of Cu-bulk and is shifted to higher binding energy. It gradually shifts with the size of the clusters, similar to the X-ray induced valence band spectra of free metal clusters. ${ }^{31}$ The very smooth spectrum of $\mathrm{Cu}_{50}$ suggests a somewhat higher coverage density for this particular sample. This is also the reason for the enhanced intensity below $5 \mathrm{eV}$ in the non-subtracted spectrum (left panel). Nevertheless the spectral shape and shift of $\mathrm{Cu}_{50}$ fit in the series of cluster spectra and suggest that the coverage density of $\mathrm{Cu}_{50}$ is low enough to maintain the individual property of the cluster. 


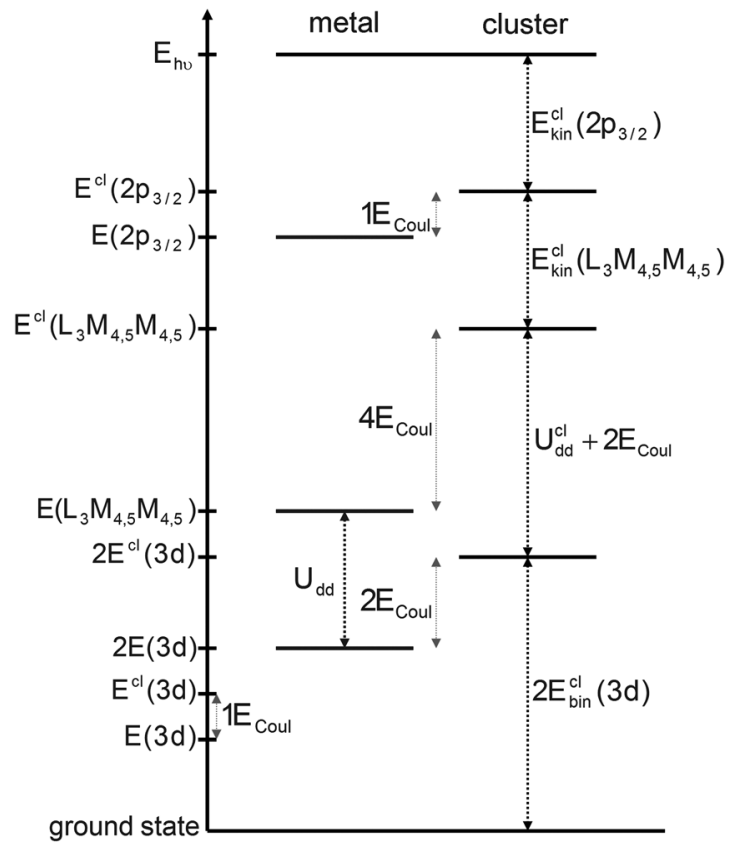

Fig. 5 Final-state energy diagram for a bulk metal and Coulomb-charged cluster used to derive eqn (2). For details see the text.

The relevant final state energies for $\mathrm{Cu}$-clusters and the bulk metal are schematically sketched in Fig. 5 on a total energy scale. The absorption of the photon increases the total energy by $h \nu$, whereby only the core hole energy remains in the system. Upon Auger decay the final state energy is reduced by leaving two $3 \mathrm{~d}$ valence holes in the system. This is compared to twice the $3 \mathrm{~d}$ photoionization energy of the valence band to obtain an estimate of the two-hole correlation energy $U_{\mathrm{dd}}$.

Upon self-convolution of the singe-hole spectral density of the $3 \mathrm{~d}$ spectrum the $\mathrm{d}-\mathrm{d}$ two-hole binding energy is obtained. The self-convoluted spectra are shown in the right panel of Fig. 4. Note that the smoothing of the spectra results from the self-convolution itself. The maximum of the self-convoluted spectra is shifted by $0.4-1.5 \mathrm{eV}$ to higher energy as compared to the bulk. Subtracting the peak energy of the two-hole spectral density and the main Auger energy from the XPS energy the sum of the two-hole Coulomb interaction energy $U_{\mathrm{dd}}^{\mathrm{cl}}$ and $2 E_{\text {Coul }}$ is derived according to eqn (2). This value is plotted versus cluster size in Fig. 6. The upper curve shows the full Coulomb interaction energy if no final-state Coulomb charging of the photoionized clusters occurs $\left(E_{\mathrm{Coul}}=0 \mathrm{eV}\right)$, i.e. when the photoionized cluster is instantly neutralized by the substrate. In this case the Coulomb interaction $U_{\mathrm{dd}}^{\mathrm{cl}}$ of two $3 \mathrm{~d}$ holes is 1-2.5 eV larger than the bulk value. The lower limit of $U_{\mathrm{dd}}^{\mathrm{cl}}$ has been calculated for a maximum final state Coulomb shift of the supported cluster. We approximate this value by the total XPS shift, $\triangle \mathrm{XPS}$, i.e. assuming that the measured binding energy shift between the cluster and the bulk is totally governed by final state Coulomb interaction between the core hole and the photoelectron $\left(E_{\text {Coul }}=\Delta \mathrm{XPS}\right)$. For that case $U_{\mathrm{dd}}^{\mathrm{cl}}$ is still somewhat enhanced with respect to the metal $(\sim 0.5 \mathrm{eV})$. In fact we assume that the Coulomb interaction energy of the deposited

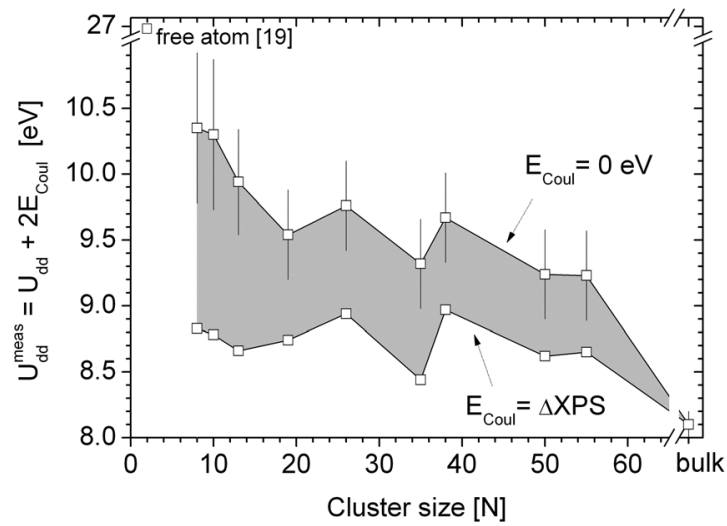

Fig. 6 The measured two-hole Coulomb interaction energy $U_{\mathrm{dd}}^{\text {meas }}$ of supported $\mathrm{Cu}_{N^{-}}$-clusters as a function of cluster size $N$. The calculated value $U_{\mathrm{dd}}$ of the free atom has been taken from ref. 19. For details see the text.

clusters resides somewhere in between the upper and lower curves. We note that the two limiting values of $U_{\mathrm{dd}}^{\mathrm{cl}}$ converge with increasing size. This shows that the final state Coulomb interaction becomes less important for larger, metallic-like particles where the extra charge is instantly screened on a timescale relevant for photoemission as in the bulk metal.

Finally, Fig. 6 exhibits that the Coulomb interaction of small supported clusters is strongly reduced with respect to the atomic value $\left(27 \mathrm{eV}^{19}\right)$, possibly due to extraatomic screening and/or polarisation by the coordinated $\mathrm{Cu}$-atoms. Moreover hybridisation among the d-orbitals of the coordinated $\mathrm{Cu}$-atoms in a cluster leads to delocalization of the charges and attenuation of the Coulomb interaction as compared to a free atom. The enhancement of $U_{\mathrm{dd}}^{\mathrm{cl}}$ with respect to the bulk phase, on the other hand, indicates that the $\mathrm{d}$-orbitals of small $\mathrm{Cu}_{N}$-clusters are more localised than in the bulk. This also explains the attenuated electrostatic screening properties of deposited $\mathrm{Cu}_{N}$-clusters as previously shown by us using PCI-Auger spectroscopy. ${ }^{18}$

\section{Summary}

Core photoelectron spectra (Auger $\mathrm{L}_{3} \mathrm{M}_{4,5} \mathrm{M}_{4,5}$, and XPS 2p, 3d) of small mass-selected $\mathrm{Cu}_{N^{-}}$-clusters $(N=8-55$ atoms) on a thin oxide film of a Si-wafer have been measured and compared to the metallic bulk phase using a dipole synchrotron beamline at BESSY. The kinetic energy as well as the XPS binding energy varies with size. While the Auger energy difference between the metallic bulk phase and the cluster amounts to $1.5-3 \mathrm{eV}$ the XPS shift of the $2 \mathrm{p}_{3 / 2}$ main peak varies between 0.3 and $0.9 \mathrm{eV}$ $(N=8-55)$. Additionally the $3 \mathrm{~d}$ valence band has been derived from the difference spectrum between the pristine $\mathrm{SiO}_{2} / \mathrm{Si}$ substrate and the cluster sample. Using the energy of the Auger main line, the corresponding core hole peak and the centroid of the self-convoluted $3 \mathrm{~d}$ valence band the Coulomb interaction energy $U_{\mathrm{dd}}$ of two d-holes as a function of cluster size has been deduced which amounts to 8.5-10.5 eV depending on the Coulomb charge of the deposited clusters. Even for the smallest clusters measured, which only contain 8 atoms, this Coulomb 
interaction is fairly reduced and only amounts to about $1 / 3$ of the atomic value while the Coulomb interaction is enhanced with respect to the bulk by about $1 \mathrm{eV}$.

\section{Acknowledgements}

Valuable discussions with G.K. Wertheim are gratefully acknowledged.

\section{References}

1 S. B. DiCenzo, S. D. Berry and E. H. Hartford, Phys. Rev. B: Condens. Matter Mater. Phys., 1988, 38, 8465.

2 C. Brechignac, M. Broyer, Ph. Cahuzac, G. Delacretaz, P. Labastie, J. P. Wolf and L. Wöste, Phys. Rev. Lett., 1988, 60, 275 .

3 W. Eberhardt, P. Fayet, D. M. Cox, Z. Fu, A. Kaldor, R. Sherwood and D. Sondericker, Phys. Rev. Lett., 1990, 64, 780; W. Eberhardt, P. Fayet, D. M. Cox, Z. Fu, A. Kaldor, R. Sherwood and D. Sondericker, Phys. Scr., 1990, 41, 892.

4 H. R. Siekmann, B. Wrenger, E. Holub-Krappe, C. Pettenkofer and K. H. Meiwes-Broer, Z. Phys. D: At., Mol. Clusters, 1993, 26, 54 .

5 H. V. Roy, P. Fayet, F. Patthey and W. D. Schneider, Phys. Rev. B: Condens. Matter Mater. Phys., 1994, 49, 5611.

6 J. O'Shea, J. Schnadt, S. Andersson, L. Patthey, S. Rost, A. Giertz, B. Brena, J.-O. Forsell, A. Sandell, O. Björneholm, P. A. Brühwiler and N. Martensson, J. Chem. Phys., 2000, 113, 9233.

7 K. Fauth, M. Heßler, D. Batchelor and G. Schütz, Surf. Sci., 2003, 529, 397.

8 S. Peredkov, S. L. Sorensen, A. Rosso, G. Öhrwall, M. Lundwall, T. Rander, A. Lindblad, H. Bergersen, W. Pokapanich and S. Svensson, Phys. Rev. B: Condens. Matter Mater. Phys., 2007, 76, 081402.

9 S. Peters, S. Peredkov, M. Neeb, W. Eberhardt and M. Al-Hada, Surf. Sci., 2013, 608, 129.

10 P. Piseri, T. Mazza, G. Bongiorno, C. Lenardi, L. Ravagnan, F. Della Foglia, F. Di Fonzo, M. Coreno, M. D. Simone, K. C. Prince and P. Milani, New J. Phys., 2006, 8, 136.

11 J. T. Lau, J. Rittmann, V. Zamudio-Bayer, M. Vogel, K. Hirsch, Ph. Klar, F. Lofink, T. Möller and B. v. Issendorff, Phys. Rev. Lett., 2008, 101, 153401.

12 S. Peredkov, A. Savci, S. Peters, M. Neeb, W. Eberhardt, H. Kampschulte, J. Meyer, B. Hofferberth, F. Menges and
G. Niedner-Schatteburg, J. Electron Spectrosc. Relat. Phenom., 2011, 184, 113.

13 P. A. Montano, G. K. Shenoy and E. E. Alp, Phys. Rev. Lett., 1986, 56, 2076.

14 S. Vajda, M. Pellin, J. Greeley, C. Marshall, L. Curtiss, G. Ballentine, J. Elam, S. Catillon-Mucherie, P. Redfern, F. Mehmood and P. Zapol, Nat. Mater., 2009, 8, 213.

15 S. Peredkov, M. Neeb, W. Eberhardt, H. Kampschulte, J. Meyer, M. Tombers and G. Niedner-Schatteburg, Phys. Rev. Lett., 2011, 107, 233401.

16 M. Niemeyer, K. Hirsch, V. Zamudio-Bayer, A. Langenberg, M. Vogel, M. Kossick, C. Ebrecht, K. Egashira, A. Terasaki, T. Möller, B. V. Issendorff and T. Lau, Phys. Rev. Lett., 2012, 108, 057201.

17 T. Lau, A. Föhlisch, R. Nietubyc, M. Reif and W. Wurth, Phys. Rev. Lett., 2002, 89, 57202.

18 S. Peters, S. Peredkov, B. Balkaya, N. Ferretti, M. Neeb and W. Eberhardt, Phys. Chem. Chem. Phys., 2010, 12, 9867.

19 E. Antonides, E. C. Janse and G. A. Sawatzky, Phys. Rev. B: Solid State, 1977, 15, 1669.

20 J. Ghijsen, L. H. Tjeng, J. Van Elp, H. Eskes and M. T. Czyzyk, Phys. Rev. B: Condens. Matter Mater. Phys, 1988, 38, 11322.

21 J. C. Fuggle, P. Bennett, F. U. Hillebrecht, A. Lenselink and G. A. Sawatzky, Phys. Rev. Lett., 1982, 49, 1787.

22 H. W. Haak, G. A. Sawatzky and T. D. Thomas, Phys. Rev. Lett., 1978, 41, 1825.

23 S. Peters, S. Peredkov, B. Balkaya, N. Ferretti, A. Savci, A. Vollmer, M. Neeb and W. Eberhardt, Rev. Sci. Instrum., 2009, 80, 125106.

24 S. Poulston, P. M. Parlett, P. Stone and M. Bowker, Surf. Interface Anal., 1996, 24, 811.

25 G. K. Wertheim, Phys. Rev. B: Condens. Matter Mater. Phys., 1987, 36, 9559.

26 W. A. de Heer and P. Milani, Phys. Rev. Lett., 1990, 65, 3356 and references therein.

27 P. H. Citrin, G. K. Wertheim and Y. Baer, Phys. Rev. B: Condens. Matter Mater. Phys., 1983, 27, 3160.

28 G. K. Wertheim, S. B. DiCenzo and S. E. Youngquist, Phys. Rev. Lett., 1983, 51, 2310.

29 T. P. Cheung, Surf. Sci., 1984, 140, 151.

30 H. Hövel, B. Grimm, M. Pollmann and B. Reihl, Phys. Rev. Lett., 1998, 81, 4608.

31 K. Jänkälä, M. H. Mikkelä and M. Huttula, J. Phys. B: At., Mol. Opt. Phys., 2011, 44, 105101. 